\title{
Co-activation of soluble and particulate guanylate cyclase by BAY 58-2667 and BNP enhances cardiorenal function in experimental heart failure
}

\author{
Guido Boerrigter*1, Lisa C Costello-Boerrigter ${ }^{1}$, Harald Lapp ${ }^{2}$, Johannes- \\ Peter Stasch ${ }^{3}$ and John C Burnett Jr ${ }^{1}$
}

\author{
Address: ${ }^{1}$ Cardiorenal Research Laboratory, Cardiovascular Research, Mayo Clinic and Mayo Clinic College of Medicine, Rochester, MN, USA, \\ ${ }^{2}$ Helios-Klinikum Wuppertal, Wuppertal, Germany and ${ }^{3}$ Cardiovascular Research, Bayer HealthCare AG, Wuppertal, Germany \\ Email: Guido Boerrigter* - boerrigter.guido@mayo.edu \\ * Corresponding author
}

from 2nd International Conference of cGMP Generators, Effectors and Therapeutic Implications

Potsdam, Germany, 10-12 June, 2005

Published: 16 June 2005

BMC Pharmacology 2005, 5(Suppl I):P5 doi:10.1 I86/I47I-22I0-5-SI-P5

\section{Background}

Heart failure (HF) is frequently associated with renal dysfunction. Thus, therapeutic strategies in HF should not adversely affect renal function. Cyclic guanosine monophosphate cGMP) is a second messenger generated by both soluble and particulate guanylate cyclase (sGC and pGC, respectively). We hypothesized that cGMP activation of sGC, pGC, or both (by BAY 58-2667, a novel direct nitric oxide-independent sGC activator [1], and/or the pGC stimulator B-type natriuretic peptide (BNP)) would have beneficial renal actions in experimental heart failure.

\section{Methods}

Heart failure was induced in 3 groups of dogs $(\mathrm{N}=7)$ by tachypacing. After 10 days, cardiorenal parameters were measured at baseline and with two intravenous doses of BAY 58-2667 (0.1 and $0.3 \mathrm{~g} / \mathrm{kg} / \mathrm{min}$ ) or BNP (10 and 50 $\mathrm{ng} / \mathrm{kg} / \mathrm{min}$ ) alone or combined. Cardiorenal function was compared within groups by analysis of variance, while groups were compared qualitatively.

\section{Results}

BAY 58-2667, BNP, and the combination of both decreased cardiac pre- and afterload and renal perfusion pressure. BAY 58-2667 alone and in combination with BNP increased cardiac output and decreased renal vascular resistance, while BNP alone did not. All three strategies increased renal blood flow. While BAY 58-2667 alone did not change urine flow, urinary sodium excretion, or glomerular filtration rate, these parameters increased with additional pGC activation. BNP alone increased filtration fraction, as did the combination with BAY 58-2667. BAY 58-2667 alone and with BNP decreased proximal fractional sodium reabsorption. There was no further activation of the renin-agiotensin-aldosterone system.

\section{Conclusion}

Direct sGC and pGC stimulation results in beneficial cardiac unloading actions in experimental heart failure, but only sGC activation increased cardiac output. Despite reductions in renal perfusion pressure, sGC activation maintained renal function, while pGC activation enhanced it. Interestingly, pGC activation increased filtration fraction and glomerular filtration rate, suggesting that it increases effective filtration pressure and/or the coefficient of filtration, whereas sGC activation does not. These findings suggest that both sGC and pGC activation do not adversely affect renal function in experimental heart failure and that co-activation may be a beneficial strategy in the treatment of heart failure to enhance cardiorenal function.

\section{References}

I. Stasch JP, Schmidt P, Alonso-Alija C, Apeler H, Dembowsky K, Haerter M, Heil M, Minuth T, Perzborn E, Pleiss U, Schramm M, Schroeder W, Schröder H, Stahl E, Steinke W: NO- and haem-independent activation of soluble guanylyl cyclase: molecular basis and cardiovascular implications of a new pharmacological principle. BrJ Pharmacol 2002, 136:773-83. 